\title{
Políticas Públicas de Ciência, T ecnologia e Inovação: possíveis contribuições para uma governança responsável da nanotecnologia no Brasil
}

Paulo F.C. Fonseca ${ }^{1}$

\section{Resumo}

$\mathrm{O}$ artigo discute algumas das principais abordagens propostas para uma governança responsável da ciência, tecnologia e inovação e, em especial, um levantamento sobre aquelas que têm se destacado nos discursos sobre políticas públicas de e para a nanotecnologia. Dentre as propostas classificadas dentro de um paradigma de políticas de ciência e tecnologia para a coesão social, se destacam aquelas relacionadas com a avaliação à montante do desenvolvimento tecnológico e a participação alargada na tomada de decisões sociotécnicas, que podem ser reconhecidas como possíveis caminhos para um desenvolvimento contra-hegemônico da nanotecnologia. Além da apresentação de alguns dos pontos centrais de abordagens que reiteram o compromisso com uma pesquisa e inovação responsável, são discutidas algumas de suas críticas e limitações. Ainda que teoricamente promissoras, tais abordagens não devem ser vistas como automaticamente transferíveis para o contexto da nanotecnologia desenvolvida no Brasil, cuja demandas e características socioculturais requerem uma reflexão situada sobre quais mecanismos institucionais podem ser viabilizados e socialmente acordados.

Palavras-chave: Políticas Públicas de Ciência, Tecnologia e Inovação; Governança; Pesquisa e Inovação Responsável; Nanotecnologia 


\section{Science, T echnology and Innovation Policy: possible contributions for a responsible governance of nanotechnology in Brazil}

\section{Abstract}

The paper discusses some of the main approaches to a responsible governance of Science, technology and innovation, stressing those that have been commonly incorporated in nanotechnology policy discourses. Within the proposals that may be acknowledged as part of The Social Cohesion Policy Paradigm in Science and Technology Policy, one may stress those concerned with an upstream technology assessment and a wide-spread public participation on sociotechnical decision making. These shall be understood as possible paths for a counter-hegemonic development of nanotechnology. The discussion encompasses some of the major tenets of the approaches that reiterate a commitment with responsible research and innovation, as well as some of their recognized critics and limitations. Even though these approaches are theoretically encouraging, they are not automatically transferable to the Brazilian environment of nanotechnology development. The Brazilian context presents distinct sociocultural features and conditions that call for a situated reflection upon the feasibility of specific institutional mechanisms, considering their potential of being socially anchored.

Keywords: Science, Technology and Innovation Policy; Governance; Responsible Research and Innovation; Nanotechnology 


\section{Introdução}

O reconhecimento dos aspectos contingenciais e situados do conhecimento científico pelos Estudos Sociais da Ciência e Tecnologia (doravante ESCT) traz a necessidade de se adotar uma postura crítica em relação às diferentes abordagens para a gestão da ciência e tecnologia. Se por um lado a ciência não é neutra, muito menos podemos admitir um desenvolvimento autônomo e linear da tecnologia, faz-se necessário orientar as redes envolvidas com a produção de fatos e artefatos tecnocientíficos de acordo com valores que são socialmente acordados, isto é, eticamente sustentados. A sociedade contemporânea, conforme aponta Ulrich Beck (Beck, 1992), é marcada pelo risco inerente à sua modernização. Mesmo as sociedades de capitalismo avançado têm sido compelidas a reconhecer a incerteza que acompanha a contínua reconfiguração dos sistemas sociotécnicos acarretada pela emergência de inovações tecnológicas. Para isso, faz-se necessário elaborar e adotar mecanismos de governança que, conforme coloca Jasanoff (2003), reconheçam humildemente a necessidade de avaliar continuamente questões éticas e sociais associadas ao desenvolvimento tecnocientífico.

Ainda que a governança não se exerça apenas pela intervenção estatal, mas pela coprodução de interações em diferentes níveis e dimensões, o Estado e as políticas públicas exercem papel crucial para a criação e estabilização de regimes sociotécnicos. A identidade, as instituições, os discursos e representações da ciência e tecnologia em um determinado contexto sociohistórico estão intrinsicamente associados às políticas públicas que buscam conformar estas dimensões de acordo com uma epistemologia específica (Velho, 2011). Este artigo, elaborado a partir de revisão de literatura e da experiência do autor com investigações no campo, irá discutir sobre algumas das distintas epistemologias que podem orientar a elaboração de políticas públicas de ciência e tecnologia e, mais especificamente, para um desenvolvimento contra-hegemônico da nanotecnologia no contexto brasileiro. 
Na próxima seção apresentar-se-ão brevemente dois paradigmas de Políticas de Ciência, Tecnologia e Inovação (PCTI), a fim de situar quais foram as propostas selecionadas para esta discussão. A terceira seção expõe os fundamentos que norteiam as propostas de pesquisa e inovação responsável, em especial o que se propõe como governança antecipatória da nanotecnologia (Barben et al., 2009). Em seguida, são discutidas algumas das críticas e limitações dessas abordagens, bem como elas podem ser consideradas no contexto da governança da nanotecnologia brasileira.

\section{Em busca de modelos alternativos de governança: paradigmas de PCTI}

É possível apontar o trabalho de alguns autores preocupados em desenvolver uma governança contra-hegemônica da ciência ou, como colocam Nunes e Gonçalves (2001), apropriar a ciência como um saber cosmopolita, "suscetível de servir para a capacitação e para a mobilização de energias emancipatórias e contra-hegemônicas" (Nunes e Gonçalves, 2001, p. 16).

Sobre essa questão, tem grande contribuição a definição de paradigmas de políticas públicas apontadas por Cozzens et al. (2008). Eles sugerem dois paradigmas para as PCTI. O primeiro é denominado Paradigma de Política para a Economia do Conhecimento - PPEC, que tem objetivos focados nos impactos econômicos da inovação. Esse modelo parte do consenso gerado em torno das análises econômicas que seguem a proposição de Schumpeter (1947), apontando para a centralidade da inovação tecnológica para a competitividade das empresas e consequentemente para o crescimento econômico nacional em meio às dinâmicas de comércio internacional (Lundvall, 2004; Freeman, 2004[1984]). A governança, nesse âmbito, se encarrega basicamente de propiciar um ambiente favorável ao investimento e desenvolvimento de inovações tecnológicas de processos e produ- 
tos, proporcionando um aumento de produtividade, a criação de novos mercados e a elevação da competitividade das empresas (Pavitt, 1984; Freeman e Soete, 2000; Lundvall e Borras, 2005). Portanto, o desenvolvimento social, para essa concepção, deve ocorrer indiretamente a partir do desenvolvimento econômico, por meio da melhoria da qualidade de produtos e serviços, pelo aumento do acesso ao emprego e assim por diante. Essa é uma visão que reduz a análise do impacto do conhecimento tecnocientífico ao campo econômico e tende a ignorar o papel direto exercido pelas inovações para a redução ou para a ampliação das desigualdades sociais (Hagendijk, Healey et al., 2009). Trata-se, portanto, do paradigma hegemônico de PCTI, o qual tem sido predominantemente adotado por países centrais e seguido pelos periféricos, incluindo as políticas públicas brasileiras das últimas décadas (Viotti, 2008; Dias, 2009).

Por outro lado, no segundo enquadramento, denominado Paradigma de Política para a Coesão Social - PPCS, os objetivos ultrapassam o "mero" desenvolvimento econômico propiciado pelas inovações para abordar diretamente questões relacionadas à inclusão social e à sustentabilidade ambiental. Coesão social, compreendida como a medida em que a sociedade trabalha conjuntamente em direção à inclusão, integração e oportunidade para todos, depende basicamente da redução das desigualdades sociais. Esse paradigma não exclui o crescimento econômico dos objetivos das PCTI, mas sugere que é possível, em diversas atribuições, como no incentivo à pesquisa básica, na articulação da interação universidade-empresa ou na regulação da propriedade intelectual, ter as preocupações com a distribuição equitativa de custos e benefícios sociais em primeiro plano. Esse paradigma representa as alternativas de governança contra-hegemônicas, que têm sido recorrentemente desconsideradas, ou relegadas a papéis figurativos ou simbólicos entre as opções políticas globais.

Cozzens et al. (2008) sugerem a necessidade de uma mudança dos pressupostos fundamentais das PCTI, caracterizando-a 
como um deslocamento do primeiro para o segundo paradigma, no qual as questões da distribuição social dos benefícios e custos da CTI são centrais. Este trabalho, da mesma forma, parte desse posicionamento de que, mais que incentivar acriticamente o surgimento de inovações, a PCTI deve atuar diretamente para a promoção de uma ciência e tecnologia com coesão social.

Assim, em consonância com as necessidades de políticas que atuem para a redução da desigualdade social e para o desenvolvimento sustentável, é preciso alertar para a forma como o primeiro paradigma vem sendo acriticamente adotado pela maioria dos países periféricos e semiperiféricos, independentemente de suas necessidades e realidades institucionais (Dagnino, 2009).

Portanto, discutir-se-ão neste espaço algumas das propostas relativas a uma governança da Ciência, Tecnologia e Inovação (CTI) que tenham a direção condizente com o PPCS, isto é, quando os objetivos políticos vão para além do crescimento econômico. Tais propostas têm apresentado materialização mais significativa dentre as novas políticas para a P\&D em nanotecnologia, denominadas por alguns como governança antecipatória (Barben, Fisher et al., 2008), mas que têm se consolidado com seu centro de gravitação no conceito de pesquisa e inovação responsável (Owen, Macnaghten et al., 2012). Trata-se de um conjunto de proposições normativas institucionais que tem sido desenvolvido especialmente por autores oriundos de países de capitalismo avançado do Norte global, que tem trazido contribuições dos ESCT para a discussão sobre a governança de tecnologias emergentes. 0 jargão Responsible Research and Innovation tem tido forte penetração no discurso institucional das agências governamentais dos países centrais europeus e dos Estados Unidos ao se referirem à emergência da nanotecnologia (Hoven, Jacob et al., 2013). Os ESCT têm apresentado uma reflexão sistemática sobre os processos de coprodução da ciência, tecnologia e sociedade, elevando a necessidade de se desenvolverem mecanismos democráticos de governança que busquem minimizar os impactos 
negativos da emergência de novas inovações. Essas abordagens fundamentam-se na necessidade de incrementar a democracia nos processos de decisão sobre questões técnico-científicas, nomeadamente através da inclusão de saberes, demandas e anseios apresentados por grupos sociais historicamente excluídos. A discussão sobre a governança responsável da nanotecnologia, neste sentido, pode ser vista como uma manifestação de vozes do "Sul dentro do Norte" (Santos, 2006), ainda que, como veremos, estas vozes possam estar sendo cooptadas pelo discurso hegemônico, servindo de legitimação política das velhas práticas de governança.

\section{Inovação Responsável e Governança Antecipatória da Nanotecnologia}

O debate político sobre a emergência da nanotecnologia tem sido marcado, em ambos os lados do Atlântico Norte, por uma preocupação em se promover um desenvolvimento responsável das inovações neste campo (European Commission, 2002; 2004; 2005; Fisher e Mahajan, 2006; Responsible Nanocode Founding Partners, 2008; Roco, Harthorn et al., 2010; Hoven, Jacob et al., 2013). Apesar do emprego do termo "responsabilidade" não ser inédito no debate sobre a ética da atividade científica (Butts, 1948; Galston, 1972), ele tem ganhado uma notória centralidade no discurso acadêmico para a governança da nanotecnologia (Royal Society e Royal Academy of Engeneers, 2004; Davies, Macnaghten et al., 2009; Robinson, 2009; Owen e Goldberg, 2010; Patra, 2011). Se por um lado o ideal de uma atuação responsável é um senso comum, não apenas na ciência, mas em todas as atividades profissionais, a definição do significado da expressão "Pesquisa e inovação responsável"2 tem sido objeto de amplo debate (Owen, Stilgoe et al., 2013; Ribeiro, Smith et al., 2016). 
Conforme apontam Stilgoe et al. (2013), a noção está presente nas concepções clássicas de uma ciência neutra e a serviço da humanidade, colocando a responsabilidade dos cientistas em se produzir conhecimento confiável, isto é, simplesmente não trapacear. Para isso, o cientista clássico responsável é aquele que consegue isolar a sua atividade de quaisquer interferências sociais e políticas, na medida em que estas poderiam comprometer a fiabilidade do conhecimento gerado, em outras palavras, o cientista responsável é aquele que negligencia a sociedade.

Evidentemente, essa concepção de uma ciência como uma república isolada (Polanyi, 1962) se choca com a compreensão da ciência e tecnologia enquanto práticas sociais e políticas desenvolvidas no âmbito dos ESCT. A ciência não é desenvolvida isoladamente, regida apenas por indagações técnicas e naturalistas, ao mesmo passo em que as tecnologias não emergem autonomamente, não há um canal linear que as empurra desde as descobertas científicas ou as puxa desde as demandas do mercado. Ao contrário de qualquer determinismo, social ou tecnológico, as novas tecnologias emergem como coprodução de interações sociotécnicas (Pinch e Bijker, 1989; Bijker, 1995). Assim, este discernimento de que as tecnologias são uma construção social, assim como as sociedades são construções tecnológicas (Thomas, 2009), implica no reconhecimento da necessidade de se governar a tecnociência de forma mais apropriada, isto é, buscar fomentar as interações mais desejáveis (Kooiman, 2003). Ao mesmo tempo, admitir que o conhecimento científico não é uma "fronteira interminável", que leva a uma compreensão fiel e universal da natureza e da sociedade, mas pelo contrário, pode paradoxalmente ampliar o nosso senso de ignorância e incerteza (Callon, Lascoumes et al., 2009), além de sugerir, não apenas uma imprevisibilidade de impactos negativos, mas também a grande probabilidade de que eles aconteçam (Beck, 1992).

Segundo Stilgoe et al. (2013), o reconhecimento de que a governança automática dos mercados não é suficiente tem leva- 
do à introdução progressiva de uma abordagem que chamam de um enquadramento consequencialista da responsabilidade. Basicamente, busca-se avaliação dos impactos de tecnologias já desenvolvidas e prontas para serem comercializadas, por um lado, e a regulamentação de questões associadas aos riscos "inexoráveis" destes produtos, por outro. No entanto, a experiência social e política tem mostrado o fracasso dessas abordagens e a literatura dos ESCT tem apontado diversos motivos para isto. 0 principal deles está associado à própria dinâmica dos processos de inovação, que costuma se caracterizar por processos de dependência das trajetórias de desenvolvimento tecnológicas, isto é, uma vez percorrido todo o caminho de desenvolvimento de uma determinada tecnologia ou sistema técnico, é muito difícil voltar atrás. Um corpo robusto de estudos tem mostrado como as inovações são marcadas por pontos de não-retorno, devido a constrições técnicas, econômicas, sociais, políticas e culturais (Dosi, 1982; Van Merkerk e Robinson, 2006). Um dos casos mais conhecidos é a permanência dos teclados QWERTY. Desenvolvido para que os martelos das máquinas de escrever mecânicas não se obstruíssem, esse padrão foi mantido mesmo após a irrelevância desta configuração para a digitação em máquinas de escrever elétricas e em teclados de computadores e telefones (David, 1985). Conforme discutiremos a seguir, o campo da avaliação de tecnologias, neste sentido, se confronta necessariamente com o que ficou conhecido como o dilema de Collingridge (Collingridge, 1980), isto é, quanto mais tarde se buscar mudar a trajetória de desenvolvimento tecnológica, mais difícil será a tarefa, mas quanto mais cedo se buscar interferir na trajetória, menos informações e previsibilidade estarão disponíveis para fazê-lo.

Por outro lado, a observação de controvérsias relacionadas com questões técnico científicas, como a energia nuclear e a biotecnologia, tem mostrado que a insatisfação ou pressão popular raramente pode ser reduzida à questão do tratamento dos riscos, mas estão também relacionadas com os próprios objetivos 
e motivações das investigações (Grove-White, Macnaghten et al., 2000). Por exemplo, a energia nuclear foi confrontada não apenas pelos riscos de possíveis acidentes como Chernobyl ou Three Miles Island, mas por fatores como a vinculação deste sistema sociotécnico com a indústria e propósitos militares e a falta de participação dos cidadãos na definição de políticas correlatas (Nelkin e Fallows, 1978; Wynne, 2011). Da mesma forma, grande parte da oposição popular aos transgênicos se dá, mais que pelo receio de que os alimentos obtidos a partir desta tecnologia possam ser prejudiciais à saúde ou ao ambiente, pela contestação à imposição do controle da agricultura global por grandes corporações internacionais privadas (Shiva, 1999; Joly, 2012).

Portanto, o conceito de governança para um desenvolvimento responsável que tem sido construído entre os analistas dos ESCT tenta ir além das limitações dessa visão "consequencialista". Para isso, existem basicamente dois desafios centrais que estruturam a maioria das abordagens: a avaliação antecipada dos impactos e a participação democrática nos processos de decisão. Nesse sentido, uma das melhores definições para a inovação responsável, por sua simplicidade, coerência e abrangência com esses dois mandatos da governança, é a apresentada por Stilgoe et al. (2013, p. 3): “Inovação responsável significa cuidar do futuro por meio de uma liderança coletiva da ciência e da inovação no presente" (Stilgoe et al., 2013, p. 3).

Essa é a essência dessa abordagem que identificamos aqui como alinhada ao PPCS e que oferece uma grande contribuição para um pensamento contra-hegemônico para a governança da nanotecnologia e da tecnociência em geral. É interessante notar como ela reverbera a missão da sociologia das ausências e das emergências proposta por Boaventura de Sousa Santos, na medida em que busca "expandir o presente" e "contrair o futuro" (Santos, 2006), isto é, reconhece-se a necessidade de, por meio de uma atuação coletiva no presente, trazer o futuro para dentro das reflexões normativas. 
Nesse sentido, a nanotecnologia tem se mostrado como uma grande oportunidade para a atuação sob essa perspectiva, já que este é um campo ainda emergente e, portanto, suscetível a uma atuação efetiva das ciências sociais (Macnaghten, Kearnes et al., 2005). Para esse objetivo, Barben et al. (2008) advogam a necessidade de se desenvolver uma "Governança Antecipatória da Nanotecnologia”, sugerindo que, ao contrário de uma metodologia específica, a promoção de uma inovação responsável deve se sustentar na coordenação das interações sociotécnicas, a partir de metodologias sustentadas pelo tripé: antecipação, participação e integração. 0 primeiro ponto se refere à necessidade de se anteciparem as questões sensíveis, sociais, econômicas, culturais e ambientais, que podem emergir com as novas tecnologias. Nesse âmbito, sugerem que é preciso promover e alargar as atividades de avaliação à montante da tecnologia, isto é, uma avaliação que não ocorra apenas $a$ posteriori, quando as tecnologias já estão completamente desenvolvidas e prontas para adentrarem o mercado. 0 segundo ponto apresenta a necessidade de se aumentar a participação democrática nos processos de decisão, incluindo o engajamento de atores que vão para além dos peritos e elaboradores de políticas, como consumidores e cidadãos interessados, não apenas na fase downstream, isto é, a fase de comercialização e difusão das tecnologias, mas também na fase upstream, isto é, na própria definição das prioridades de investigação e políticas de fomento. Para isso, trazem ao debate a sólida evolução dos estudos sobre percepção e participação pública da ciência. 0 terceiro ponto se refere à necessidade de se integrarem considerações mais abrangentes nas próprias atividades de Pesquisa e Desenvolvimento (P\&D), que têm sido historicamente isoladas de qualquer vinculação direta com preocupações sociais ou ambientais. Para isso, ainda que os autores identifiquem a pertinência do legado dos estudos de laboratório para a compreensão e possível atuação nesse universo, a principal tarefa é justamente buscar integrar os resultados e feedbacks das duas primeiras metodologias nos próprios processos de P\&D. 
Por se tratar do cerne das discussões sobre o desenvolvimento responsável de tecnologias emergentes e, particularmente, que tem permeado o debate recente sobre a governança da nanotecnologia, vamos discutir mais detalhadamente cada um destes pressupostos metodológicos que orientam a racionalidade da governança antecipatória.

\subsection{A avaliação de tecnologias: da análise de impactos ao desenvolvimento reflexivo}

Uma das organizações de avaliação de tecnologias (AT) de maior relevância histórica é a OTA - Office of Technology Assessment, criada pioneiramente pelo Senado dos Estados Unidos em 1973 e extinta em 1995. Wood (1997) sugere que os principais motivos que levaram ao seu encerramento foram de cunho político, afirmando que faltou maior solidez para o órgão num momento de transição de prioridades e filosofia política do congresso americano. Uma das causas é que, apesar de seu estatuto e constituição bipartidária, a percepção era de que a agência servia melhor aos interesses do partido majoritário na legislatura anterior (Cope, 2002). No entanto, o debate público foi além da situação política da organização, levantando questões que influenciaram a investigação sobre métodos, gestão e objetivos da AT. Sclove (2010) aponta como os próprios congressistas ressaltaram que a boa qualidade dos estudos não podia ser aproveitada para a decisão legislativa devido à lentidão com que eram entregues os relatórios, em contraste com a pressão econômica por dinamismo do processo regulatório. Ou seja, a avaliação, feita a posteriori, não estava sendo efetivamente considerada, pois quando esta se concluía, o lobby pela regulação apropriada para a comercialização já havia obtido sucesso. Por outro lado, a comunidade dos ESCT alertou sobre como se alimentava a falsa concepção de que relatórios técnicos conduzidos por peritos podiam estar isentos de juízos de valor. Segundo Sclove (2010), a OTA falha, não por 
não conseguir ser neutra, mas por não assumir a sua inerente parcialidade na escolha dos métodos e objetivos dos estudos. Por exemplo, as rotinas de análise passavam necessariamente por aspectos como importância para o crescimento econômico e produtividade, mas nunca por questões como as implicações para qualidade do trabalho, para as relações de gênero, raça e poder das comunidades frente às grandes corporações comerciais. Assim, em sua perseguição por demonstrar objetividade, seus estudos não obtiveram sucesso em elucidar as verdadeiras implicações éticas e sociais das novas tecnologias, na medida em que nunca se buscou integrar as visões dos cidadãos comuns dentro das avaliações sobre as tecnologias.

Nesse sentido, ao mesmo tempo em que o encerramento dessa importante instituição pública de AT foi lamentado, o evento abriu espaço para reflexão e promoção de outras abordagens com finalidades públicas, nomeadamente aquelas fundamentadas em mecanismos de participação mais alargada e cujo escopo ultrapassa a análise a posteriori dos impactos de tecnologias já desenvolvidas e se propõem a atuar ao longo do processo de desenvolvimento, algo que ficou inicialmente consolidado com o conceito de Avaliação Construtiva de Tecnologias (ACT).

Schot e Rip (1997) dizem que o propósito geral da ACT é "alargar o desenho das novas tecnologias (e o redesenho das antigas)" (Schot e Rip, 1997, p. 252). Para isso, o feedback das atividades de AT na construção atual da tecnologia é crucial, e são as estratégias e instrumentos para promover este retorno que compõem o cerne da ACT. Para tanto, vão desde experimentos sociais, conferências para diálogo até programas de direcionamento tecnológico. 0 termo "construtiva" indica, por um lado, o compromisso com a atuação na construção, isto é, na utilização dos seus instrumentos para guiarem as opções de construção das novas tecnologias, mas também se refere à perspectiva construtivista da ciência e tecnologia. 
A gênese das abordagens de ACT é creditada à criação e às subsequentes iniciativas do órgão holandês para avaliação de tecnologias, o NOTA (Netherlands Office of Technology Assessment), nos anos 80 (Schot, 2001).

Segundo Schot e Rip (1997), a ACT é resultado de uma redefinição das fronteiras entre as políticas de desenvolvimento e de avaliação de tecnologias na medida em que ambas têm o objetivo comum de promover tecnologia que tenha muitos impactos positivos e poucos negativos. São diversos os métodos que, ainda que não desenvolvidos para esses propósitos, fazem parte da agenda da ACT. Por exemplo, o mapeamento social, uma espécie de análise de stakeholders envolvidos, presente em todas as AT tradicionais, como as praticadas pela OTA, deve se estender ao chamado mapeamento sociotécnico, na medida em que deve entender as dinâmicas sociais das trajetórias tecnológicas. Outra categoria, são aquelas de experimentação controlada com novas tecnologias como demonstradores sociotécnicos. No entanto, talvez o método que tem sido mais utilizado é a elaboração e discussão de cenários, isto é, são elaborados distintos cenários sobre o futuro de determinadas tecnologias, para serem discutidos por distintos stakeholders da cadeia de inovação, consumo e gestão de resíduos. Os cenários contemplam não apenas aspectos técnicos sobre a direção e configuração das futuras trajetórias de desenvolvimento tecnológico, mas as interligações destes com questões regulatórias de natureza econômica, mas sobretudo ambientais, culturais e sociais (Rip e Kulve, 2008; Robinson, 2010).

Assim, o cerne da concepção de ACT está no retorno, ou na integração desta avaliação no próprio processo. A avaliação deve ser um dos coprodutores dos impactos tecnológicos, e não apenas um estágio posterior de avaliação dos impactos produzidos.

No entanto, afirmar a importância de se participar dos processos à montante do seu desenvolvimento não é por si suficiente. 
É preciso especificar e compreender como tal participação pode ser feita. Nesse sentido, Schot e Rip (1995) afirmam que é importante que a ACT, como qualquer atividade de AT, busque não se influenciar ou privilegiar alguns dos diferentes atores envolvidos no processo de coprodução. Para isso, uma das prioridades deve ser a promoção da aprendizagem por parte de todos os atores. Isso possibilita uma efetiva ligação das questões técnicas, comerciais, políticas e culturais na medida em que abre o universo de opções e restrições de cada uma das partes. Portanto, para Schot e Rip (1995) é necessário um aprendizado profundo, não apenas sobre o trabalho e objetivos do outro, mas sobre os valores, percepções e concepções incrustradas em cada ator, seja individual ou coletivo.

Em outras palavras, o objetivo das propostas da ACT é promover a reflexividade. Os diferentes atores devem ser reflexivos sobre a coprodução da tecnologia e seus efeitos, porque é esta capacidade que pode melhorar o processo de desenvolvimento. É preciso que, não apenas os cientistas e técnicos, mas os responsáveis pelas políticas públicas, os consumidores e as comunidades relacionadas estejam cientes sobre a coprodução de ciência, tecnologia e sociedade, para que possa haver uma modulação em direção a resultados socialmente mais harmoniosos.

0 aumento dos investimentos em inovações, não apenas materiais, mas sociais e simbólicos, pode gerar irreversibilidades, "caminhos sem volta", associados às tensões geradas pelas expectativas, padrões de definição de agenda e altos investimentos (Van Merkerk e Van Lente, 2005; Van Merkerk e Robinson, 2006). Existem padrões nessas irreversibilidades, mas não existe um modelo descritivo generalizado. Rip (1995) afirma que a constatação da sociologia e economia modernas sobre a não linearidade e dependência contingencial dos processos levanta pouca esperança para a aplicação direta de modelos teóricos, mas isto não impede que tais achados sejam usados como instrumentos para as análises específicas. São as interações entre 
os diversos atores que vão determinar os resultados, não há um único ator isolado que possa de fato conduzi-las. Portanto, a questão da ACT é que sejam tomadas trajetórias irreversíveis mais desejáveis que outras. Daí a necessidade de um aprendizado para a reflexividade generalizada, para que as interações se deem dentro de uma cultura mais crítica e responsável sobre os destinos aos quais levarão determinadas trajetórias. Os tradicionais mecanismos de controle centralizado e hierarquizado não costumam ser bem sucedidos.

Assim, uma das características principais das distintas abordagens para uma governança antecipatória ou responsável da nanotecnologia e das inovações em geral é o alargamento da participação de outros atores nas próprias atividades de avaliação. Ainda que com distintos nomes, como Avaliação Participativa (KlüVer, Nentwich et al., 2000), Interativa (Marris, Joly et al., 2008), em Tempo Real (Guston e Sarewitz, 2001) ou Integrada (Forsberg, Ribeiro et al., 2016), estas abordagens podem ser vistas como partes constitutivas da crescente influência dos ESCT em sua dimensão processual, isto é, que reconhece a política inerente às decisões sociotécnicas e que, por isto mesmo, propõe mecanismos de deliberação democrática (Fonseca, 2012). Conforme bem apontam Barben et al. (2009), o clamor pela participação deliberativa se desenvolve, não só no campo da avaliação de tecnologias que acabamos de discutir, mas também a partir da tradição dos estudos sobre percepção e comunicação pública da ciência. Ainda que essa divisão disciplinar não corresponda à natureza da literatura dos ESCT e dos mecanismos e modelos propostos para participação pública neste âmbito, uma discussão específica sobre a evolução das práticas de comunicação científica tem utilidade para a clareza desta exposição. 


\subsection{V isões sobre a comunicação e compreensão pública da} ciência

Pode-se dizer que os estudos sobre comunicação e percepção pública da ciência fundamentam-se em duas concepções distintas: o modelo do déficit e da participação (Lewenstein, 2003; Wilsdon e Willis, 2004; Jasanoff, 2005). O primeiro está associado à concepção clássica e herdada da ciência enquanto conhecimento e método universal e exclusivo e, portanto, concebe a política de comunicação como uma transferência de informações desde a ciência para a sociedade. A segunda reconhece a parcialidade do conhecimento e dos atores tecnocientíficos, assim como o valor de saberes alternativos e contextuais de populações "leigas", concebendo, portanto, a política de comunicação a partir da promoção de diálogo e participação.

\subsubsection{M odelo de déficit e educação}

Difundida e desenvolvida especialmente a partir dos anos 70 como resposta ao início da perda de credibilidade sentida pela comunidade científica, essa abordagem interpreta como causa dessa crise de confiança a falta de informação sobre os reais benefícios da ciência. Assim, o significado da promoção de uma melhor compreensão pública da ciência, de acordo com essa perspectiva, é a promoção de uma melhor apreciação da ciência pelo público (Lewenstein, 1992). Num primeiro momento, a solução para controvérsias de caráter tecnocientífico sustentava-se na "constatação"3 de que o público tinha pouco conhecimento sobre a ciência, portanto um déficit, e que seria preciso melhor educar os cidadãos para que estes 
compreendessem os benefícios dos avanços tecnocientíficos e a possibilidade de uma gestão segura dos riscos. Por exemplo, o rechaço da população em relação à energia nuclear, ou mais recentemente em relação aos alimentos transgênicos, deveria ter sido causado pela desinformação da população, não apenas em relação aos reais benefícios que estas tecnologias trazem, mas também por não compreenderem a "inquestionável" fiabilidade e eficiência do conhecimento científico e suas técnicas de segurança. A solução para isto seria, de acordo com essa visão, melhorar a qualidade da comunicação com o público leigo sobre os fatos científicos, ou seja, promover uma ampla alfabetização científica. Os cidadãos, uma vez informados e capacitados ao entendimento racional do método e conhecimento científicos, deveriam apoiar o avanço da técnica e do conhecimento científico indiscriminadamente (The Royal Society of London, 1985).

Portanto, o modelo de comunicação entre as instituições científicas e a sociedade em geral, de acordo com essa perspectiva, é unidirecional, isto é, deve haver uma transferência de conhecimento desde as instituições e atores científicos para a sociedade. Pela ótica da sociologia das ausências, esse é o mecanismo básico para a ocorrência do "epistemicídio" de outros saberes válidos ao qual se refere Boaventura de Sousa Santos (Santos, 2010).

Evidentemente, buscar oferecer aos cidadãos leigos uma formação científica adequada não é suficiente para estabelecer a confiança de determinados grupos sociais nas instituições científicas, muito pelo contrário, o que se observou foi uma crescente deterioração da confiança e da credibilidade de instituições, relatórios e decisões tecnocientíficas. São diversos os casos de estudo que apontam a ineficiência desses tradicionais modelos de comunicação (Jasanoff, 2005; Jasanoff; Kim, 2009) para lidar com as insatisfações sociais em casos de controvérsia a respeito de questões tecnocientíficas. 
Ainda que seja preciso reconhecer a importância da educação e divulgação científica, estas não podem ser tomadas como suficientes para um desenvolvimento harmonioso de sistemas sociotécnicos de inovação. Nesse sentido, há também um crescente número de estudos sobre que tipo de educação científica é a mais desejável, com um grande reconhecimento de que é preciso não só ensinar sobre o método e corpo de conhecimentos científicos, mas principalmente estimular a capacidade analítica para a compreensão crítica de questões sociotécnicas (Linsingen e Cassiani, 2013). Ou seja, é preciso educar, não para moldar, mas para capacitar. No entanto, essa racionalidade tem se mantido bastante presente entre as perspectivas sobre educação e comunicação científica e, infelizmente, tem estruturado a concepção de diversas políticas públicas no Brasil.

\subsubsection{M odelo de diálogo e participação}

A segunda perspectiva surge a partir das críticas a essa primeira, reconhecendo inicialmente que os cidadãos também têm um tipo de conhecimento relevante, um tipo de expertise, associado ao contexto em que estão inseridos e a partir do qual devem lidar com determinadas questões tecnocientíficas. Assim, torna-se necessária não uma comunicação unidirecional, mas um maior diálogo e interação dos especialistas com o público leigo (Wynne, 1992; Irwin; Michael, 2003). Portanto, dentro dessa visão, a comunicação sobre ciência e tecnologia deve se dar em via de mão dupla, é preciso, além de informar, escutar, dialogar e incorporar as possíveis contribuições do público "leigo", isto é, com racionalidades desenvolvidas independentemente de treinamento tecnocientífico.

Diversos analistas apontaram ser necessária uma maior humildade da ciência em relação às próprias limitações e incertezas (Jasanoff, 2003). São recorrentes os casos históricos em que é possível observar que os próprios peritos avaliadores de ris- 
co discordam entre eles mesmos, omitem e selecionam dados de acordo com os próprios interesses ou limitações, e que julgamentos de valor são feitos em todas as etapas do manejo do risco feito de acordo com todos os protocolos de racionalidade e objetividade científica (Wynne, 1992; Rowe; Frewer, 2000). Muitas vezes, governos auxiliados por pareceres tecnocientíficos, ao contrário de resolverem as controvérsias com a evidência científica, minaram ainda mais a desconfiança das populações, tanto nos peritos como nos próprios burocratas encarregados pelas decisões governamentais. Por exemplo, a epidemia de encefalopatia espongiforme bovina, vulgarmente conhecida como doença da vaca louca ou BSE (do inglês bovine spongiform encephalopathy), foi uma controvérsia que deixou em evidência o fim do contrato social que conferia legitimidade aos peritos para decidirem em nome de toda a sociedade acerca de questões supostamente técnicas, na medida em que os peritos e os governantes não admitiram adequadamente as incertezas presentes em relação à possível transmissão para humanos, um fenômeno que Jasanoff (1997) caracterizou como "deslocação cívica".

Callon et al. (2009) identificam uma crise do modelo institucional de dupla delegação, isto é, o fim da aceitação social da delegação aos cientistas e peritos para decidirem sobre quaisquer questões que envolvem riscos de natureza tecnocientífica e da delegação a burocratas do estado para centralizarem as decisões políticas, uma crescente desconfiança em relação às próprias instituições políticas e científicas. Por outro lado, os autores sugerem que as controvérsias tecnocientíficas devem ser discutidas em espaços que eles chamam de "fóruns híbridos". Os mesmos sugerem o termo "fórum" porque são espaços abertos onde grupos podem se reunir para discutir opções técnicas que envolvem o coletivo, e "híbrido" porque os grupos envolvidos, ou os porta-vozes que alegam representar estes grupos, são heterogêneos, incluindo peritos, políticos, técnicos e pessoas leigas que se considerem envolvidas. Da mesma forma, são híbridos porque as questões e problemas são abordados por diferentes 
perspectivas e domínios, como a ética, a economia, veterinária ou física nuclear. Se por um lado eles identificam que esses fóruns têm emergido espontaneamente em domínios como a crise da BSE, dos transgênicos ou da energia nuclear, eles reforçam a confiança de que estes fóruns, baseados na experimentação e aprendizado coletivo, são uma resposta apropriada para se lidar com a crescente incerteza inerente à tecnociência.

São diversos os mecanismos ou metodologias desenvolvidos para promover a participação pública, ou o que Laurent (2011) chama de "tecnologias de democracia". Rowe e Frewer (2005) sugerem uma tipologia para os exercícios de participação que distingue entre comunicação, consulta e participação. A primeira, para o âmbito da ciência e tecnologia, tem relevância especialmente no paradigma do déficit, e consiste em informar unidirecionalmente o público. A segunda consiste em consultar, mas também unidirecionalmente, o público. Essa, no entanto, já pode ser incluída dentro da perspectiva de engajamento, na medida em que o público tem voz ativa. A terceira consiste em uma via de mão dupla, em que o público interage e participa da construção de conhecimento em conjunto com os organizadores do exercício de participação e é, evidentemente, o modelo mais desejável desde o ponto de vista democrático, mas também o mais difícil de se organizar e avaliar o sucesso. Para a participação, definem quatro tipos de mecanismos, classificados a partir das variáveis: método de seleção de participantes (controlada ou não), informação prévia fornecida aos participantes (flexível ou pré-definida), modos de resposta dos participantes (ilimitada e aberta ou limitada e fechada), meios de transferência de informações (cara a cara ou indireta), facilitação da agregação das informações geradas (estruturada ou não-estruturada). Não caberia aqui uma discussão mais aprofundada sobre cada um desses tipos, mas sim ressaltar que há uma crescente literatura sobre o desenvolvimento e avaliação destes mecanismos de deliberação democrática, não apenas no âmbito da ciência e tecnologia, mas da ciência política em geral. Nesse sentido, são con- 
soantes as discussões sobre a crise da dupla delegação e a virada deliberativa presente nas ciências políticas e nos próprios mecanismos de avaliação de tecnologias, conforme discutimos acima.

Assim, especialmente a partir dos anos 2000, há uma clara transição no discurso governamental de países como o Reino Unido, Estados Unidos e França, da expressão "compreensão pública da ciência" para "engajamento público na ciência" (Wilsdon; Willis, 2004). Nesse sentido, a nanotecnologia aparece como o palco propício para o desenvolvimento acadêmico e político de experimentações desse novo paradigma de engajamento público. Uma das abordagens que se destaca é a proposta que tem sido desenvolvida especialmente no Reino Unido, que Wilsdon e Willis (2004) chamam de Upstream Public Engagement (UPE). Trata-se de uma mudança no discurso político do New Labor vivenciada na Inglaterra a partir dos anos 2000 (Thorpe, 2010), que teve maiores materializações em exercícios relacionados com a nanotecnologia. 0 termo upstream indica que a participação pública se dê não apenas uma vez que as controvérsias se instaurem, mas sim a montante das próprias atividades de P\&D. Portanto, trata-se de uma abordagem que comparte o mesmo paradigma de responsabilidade da ACT, na medida em que advoga a participação alargada e antecipada nos processos de decisão sobre questões tecnocientíficas. Ao contrário de conceber ciência, tecnologia e sociedade separadamente, reconhece um sistema sociotécnico que requer atenção para o exercício da democracia entre os vários espaços de coprodução de conhecimento, tendo em vista os riscos da crescente tecnologização das práticas sociais e culturais inerentes à condição moderna (Beck, 1992).

Segundo Wilsdon e Willis (2004), os mecanismos relacionados com essa visão são fundamentados nas tradicionais políticas de comunicação e participação, mas sobretudo no aprendizado sobre as suas falhas. Assim, identificam que esses novos dispositivos, ao contrário de se fundamentarem apenas no conhecimento dos próprios peritos em comunicação, devem incluir outras for- 
mas mais "plurais e diversas de conhecimentos públicos que estavam sendo marginalizados" (Wilsdon; Willis, 2004, p. 22) Da mesma forma, identificam que os tradicionais modelos adotam um enquadramento para a discussão das controvérsias pela ótica restrita da análise de riscos implicados nas novas tecnologias e sugerem que um engajamento desejável deve incluir questões antes ignoradas, relacionadas com a propriedade, o controle e os fins sociais das tecnologias. Por fim, identificam que muitas das atividades prévias ocorriam em uma espécie de vácuo, isto é, não apresentavam uma conexão direta com as decisões técnico políticas, como as opções e prioridades de investigação e inovação ou com a elaboração de políticas públicas sobre inovação.

De fato, a vinculação ou a integração efetiva da participação com as decisões sociotécnicas é, ao mesmo tempo, o maior desafio e a maior condição para que estas propostas sejam bem sucedidas para promover a inovação responsável. É esse o terceiro pilar da governança antecipatória, a integração de considerações mais abrangentes dentro do próprio ambiente de P\&D. Nesse sentido, ainda que as atividades de ACT e de participação cidadã a montante só façam sentido se os seus resultados forem efetivamente integrados às decisões relacionadas com as atividades de P\&D e PCTI, existem iniciativas específicas que buscam estruturar um modelo de colaboração direta de cientistas sociais e naturais no próprio laboratório, como aquela desenvolvida no âmbito acadêmico estadunidense, denominada midstream modulation.

Trata-se de uma proposta que comparte os mesmos pressupostos e objetivos das metodologias de ACT e da participação pública a montante, isto é, busca contornar o "Dilema de Collingridge" por meio do aumento da reflexividade dos próprios desenvolvedores de inovações, evitando os recorrentes fracassos de iniciativas de controle verticalizados ou de regulação a posteriori. 


\section{0 discurso para a governança da nano nos países centrais: antecipação como legitimação?}

Não é difícil notar como essas distintas abordagens teóricas dirigidas à governança das tecnologias emergentes, que Barben et al. (2009) sugerem como Governança Antecipatória, são materializações de um mesmo paradigma, oriundo dos ESCT, que contempla uma governança mais robusta, que tem sido definida como "responsável", das inovações tecnocientíficas. Ao mesmo tempo em que reconhece a complexidade dos sistemas sociotécnicos, não se resigna em buscar uma coordenação que se fundamente em valores como democracia, justiça social e sustentabilidade ambiental. Para isso, se direciona especialmente para o ambiente de pesquisa em si, são os cientistas seu grande público alvo. Ainda que a participação cidadã seja central, o que se espera são que as práticas de pesquisa sejam de algum modo modeladas de acordo com estes valores, que os cientistas, a partir do reconhecimento dos aspectos humanos e sociais de suas decisões técnicas, exerçam o seu "poder" de forma mais responsável. A fuga para o dilema de Collingridge é, portanto, a responsabilidade. A saída para o reestabelecimento da confiança nas instituições científicas e de poder político é a participação democrática, e quando possível, deliberativa.

Porém, se por um lado esses modelos são desenvolvidos e discutidos academicamente, talvez o que chame mais atenção é a inserção desse vocabulário no recente discurso político dos países centrais no desenvolvimento da nanotecnologia. As políticas de nanotecnologia, especialmente no contexto norte-americano e europeu, têm sido notabilizadas por apresentarem uma inédita chamada para um maior diálogo e participação pública a montante da pesquisa e desenvolvimento. As iniciativas políticas desses países têm recorrentemente utilizado a expressão "pesquisa e inovação responsável", explicitamente referindo à necessidade de maior participação e antecipação dos possíveis impactos de novas tecnologias. Diversos documentos da PCTI 
britânica utilizam a expressão upstream engagement para se referir às atividades de comunicação sugeridas para as políticas de nanotecnologia. (Royal Society e Royal Academy of Engeneers, 2004; Uk Government, 2005). Nos Estados Unidos, documentos relacionados com a iniciativa nacional de nanotecnologia ressaltam a necessidade de se abordar as dimensões sociais da nanotecnologia (Roco, Harthorn et al., 2010), a própria legislação aprovada incluiu esta preocupação, e a Fundação Nacional para a Ciência tem provido um financiamento significativo para a investigação nesse âmbito. Na Holanda, seguindo já a tradição prévia, o programa nacional de nanotecnologia inclui a ACT como uma de suas atividades básicas. No entanto, talvez a entidade política em que seja mais nítida a inserção do discurso dos ESCT nos documentos estratégicos e o financiamento para atividades de antecipação e participação relacionadas com a nanotecnologia seja a Comissão Europeia. Os documentos principais da estratégia europeia para a nanotecnologia colocam explicitamente a necessidade de realizar "um desenvolvimento responsável e democrático da nanotecnologia" (European Commission, 2004; 2005), e também foram financiados diversos projetos de investigação sobre as dimensões éticas e sobre mecanismos de diálogo e participação.

No entanto, apesar do discurso acadêmico, conforme vimos, ser bastante contra-hegemônico, muitos analistas têm apontado como ele tem sido apropriado como uma política de legitimação da mesma racionalidade que tem guiado a inovação, isto é, um desenvolvimento exclusivamente motivado pelo lucro de corporações privadas.

Isso está relacionado ao fenômeno do aprendizado político e comercial_ gerado a partir do histórico de perda de credibilidade das instituições científicas e da burocracia política. É consensual que as sucessivas controvérsias observadas nos países centrais, como os Organismos Geneticamente Modificados OGM, a BSE e as crises financeiras, minaram a confiança da população 
em relação aos pronunciamentos oficiais em relação à Ciência e Tecnologia (Jasanoff, 2003). Portanto, há um claro receio de que a nanotecnologia, assim como os OGM, seja rejeitada pelos consumidores devido aos riscos inerentes aos seus produtos, algo que Rip (2006) caracterizou bem ao identificar um sentimento de "nanofobia-fobia" entre os promotores da nanotecnologia. Irwin (2006) sugere que permanece ainda uma percepção de déficit, não de conhecimento, mas de confiança da sociedade em relação à Ciência e Tecnologia e que, portanto, tais iniciativas para o diálogo e participação têm a finalidade de dirigir o público a um consenso e uma consequente aceitação dos novos produtos tecnocientíficos. Barben (2010) sugere que essa crise de confiança continua sendo tratada dentro do mesmo paradigma de "políticas de aceitação", isto é, políticas destinadas a promover a aceitação pública das inovações tecnológicas, e não para promover uma coprodução de inovações genuinamente mais democrática. Já Thorpe (2010) questiona os motivos pelos quais a resposta governamental no Reino Unido foi, ao invés de se aumentar a robustez dos tradicionais sistemas tecnocráticos de controle e regulamentação, a de promover uma ampliação do diálogo e uma democratização do acesso à perícia. Segundo ele, os motivos têm raízes no próprio modelo da "terceira via" adotado pelo partido trabalhista britânico e fortemente vinculado à estratégia de desenvolvimento da Comissão Europeia ao longo da última década (Ue, 2000; Van Apeldoorn, 2006). Baseando-se na visão de uma nova economia pós-fordista, caracterizada pela transição de uma produção, nas economias avançadas, de bens de consumo materiais para a "produção de conhecimento", os defensores desta abordagem veem esta ampliação da participação não apenas como possível, mas até necessária para incentivar a consolidação de um tipo de cidadania ativa e criativa, trabalhadores e consumidores de conhecimento que deveriam contribuir para o crescimento econômico.

A definição de desenvolvimento responsável por Von Schomberg (2011) para a comissão europeia que tem sido recorrentemente 
utilizada oferece oportunidade para reconhecer a validade destas críticas:

\begin{abstract}
Investigação e Inovação Responsável é um processo transparente e interativo no qual os atores sociais e inovadores se tornam mutualmente responsáveis de acordo com um visão sobre a aceitação (ética), a sustentabilidade e desejeabilidade social de processos de inovação e os seus produtos comercializáveis (de forma a permitir a devida difusão dos avanços científicos e tecnológicos na nossa sociedade) (Von Schomberg, 2011, p. 9).
\end{abstract}

Fica claro que o conceito propagado de responsabilidade, ainda que inclua os valores de transparência, participação, sustentabilidade e fim social, os utiliza de forma sugestivamente utilitarista, isto é, como meios para garantir a difusão dos avanços tecnocientíficos na sociedade. A colocação do parêntesis na expressão “aceitação (ética)", por outro lado, sugere que a centralidade da política de aceitação que fala Barben (2010).

Por outro lado, dentro da própria literatura gerada pela multiplicação de experiências, há uma crescente reflexão sobre até que ponto os resultados atingidos têm sido aqueles almejados pela produção acadêmica (Van Doren, Forsberg et al., 2014). Wynne coloca bem a questão ao sugerir que as instituições políticas têm tocado as notas, mas não a música (Wynne, 2006). Apesar da proliferação de estudos e propostas políticas verificadas ao longo da última década, tem sido recorrente o ceticismo em relação à viabilidade de sucesso das iniciativas de integração da avaliação e participação pública nas atividades de I\&D (Fisher, Mahajan et al., 2006; Joly; Kaufmann, 2008; Doubleday; Viseu, 2010). Em especial, ressalta-se que tais iniciativas, quando desenvolvidas, enfrentam um atrito substancial causado pela hegemonia das concepções clássicas sobre a ciência e a tecnologia, que permanece incrustada na racionalidade da comunidade de investigação e elaboração de políticas. Por exemplo, Doubleday 
e Viseu (2010) apontam que os cientistas sociais são legitimados, ora como pontes entre os cientistas e a sociedade, ora como representantes da própria, e, portanto, mantendo a mesma concepção de que o laboratório deve estar isolado das interferências externas. Da mesma forma, identificam que as assimetrias de poder entre os cientistas e peritos de um lado, e os cientistas sociais e o público leigo de outro, são problemas cujas soluções não se encontram num horizonte próximo.

Em relação ao debate sobre atividades de avaliação, o caráter sistêmico e aberto do progresso do conhecimento tecnocientífico torna a prospecção um exercício de alcance limitado, algo que reforça a resistência por parte de empresas e atores governamentais à interferência política nos processos de $\mathrm{P} \& \mathrm{D}$, obstáculos históricos à implementação de tais práticas (Guston; Sarewitz, 2001). Um dos dilemas que se tem levantado para a dificuldade de se levar a cabo esse tipo de governança é o fato de que os agentes são concomitantemente os principais, isto é, os governos são os principais interessados no rápido avanço das nanotecnologias, mas também responsáveis por segurar o passo do seu desenvolvimento face as possíveis consequências indesejáveis, o que tem gerado uma constante tensão em relação a estes dois propósitos, com a adoção de medidas que são aparentemente contraditórias (Whitman, 2007).

\section{Importando mecanismos ou importando-se com abordagens?}

As políticas de nanotecnologia no Brasil têm apresentado um isomorfismo parcial em relação às políticas dos países centrais, isto é, têm repetido as medidas sintonizadas com o PPEC, mas não têm apresentado a mesma ênfase em se promover a participação alargada e uma avaliação antecipada que busque integrar as preocupações éticas, sociais e de segurança (Invernizzi, 2011). Isso está relacionado com o contexto distinto de governança da 
ciência e tecnologia local, que se fundamenta em distintos imaginários sociotécnicos (Jasanoff; Kim, 2009) para a nanotecnologia. Nos países centrais, a nanotecnologia é um campo crucial para a manutenção da liderança no comércio global de inovações científicas, e para a manutenção desta posição foi identificada a necessidade de se evitar um rechaço da população como o que aconteceu com os OGM no contexto europeu, o que tem levado à disseminação do discurso do desenvolvimento responsável da nanotecnologia. Já no contexto semiperiférico em que se insere o Brasil, essa é vista como a grande oportunidade para se promover o deslocamento para a posição central na economia global, na medida em que trata-se de um campo emergente e, portanto, com muitas possibilidades abertas. Portanto, o imaginário sociotécnico local para a nanotecnologia não vislumbra, ao contrário dos primeiros, a possibilidade de rechaço destas pelos seus cidadãos, mas reduz a questão à necessidade de incorporá-la ao tecido industrial a fim de se incrementar a produtividade e a competitividade.

Isso se deve, por um lado, à histórica relação entre o ambiente de elaboração das políticas e a própria comunidade de investigação em ambos os contextos. Renato Dagnino (2006; 2007) identifica que, ao contrário do que tem se desenvolvido em muitos dos países centrais, não há no Brasil uma separação de papéis entre os elaboradores da PCT e a própria comunidade de investigação, isto é, os cientistas têm sido eles mesmos os policy makers. Por exemplo, para o caso específico da nanotecnologia, Santos Junior identifica como os atores presentes nos comitês responsáveis pela elaboração política são também os atores que têm se destacado como líderes na produção científica na área (Santos Junior, 2011) Assim, em conformidade com o que apontam também analistas voltados para o contexto dos países centrais (Doubleday; Viseu, 2010), Dagnino (2007) identifica que a racionalidade hegemônica entre a comunidade de investigação e, portanto, entre os próprios elaboradores de políticas, tem mantido as concepções clássicas do ethos mertoniano da ciência 
e, portanto, reproduzido uma concepção positivista, calcada na linearidade do processo de inovação e no determinismo tecnológico. Por exemplo, Dagnino (2007) sugere que a recente incorporação do paradigma da centralidade na inovação na PCTI brasileira, nomeadamente pela transferência de conhecimento academia-indústria e pelo incentivo à privatização do conhecimento científico, tem servido como legitimação e garantia dos históricos privilégios reservados a determinados setores da classe científica, nomeadamente a Física e a Química, na medida em que se mantém o status privilegiado destes enquanto propulsores do desenvolvimento econômico.

Portanto, se nos próprios países centrais essa virada para a responsabilidade tem sido vista com ressalvas, é preciso ter ainda mais cautela ao buscar implementar um paradigma semelhante em um contexto socioinstitucional distinto como o do Brasil. Assim como se argumenta para o caso das políticas de incentivo à inovação calcada no paradigma PPEC, o isomorfismo das políticas de estímulo à responsabilidade não é desejável, na medida em que é preciso reconhecer a heterogeneidade de valores culturais, demandas sociais e configurações políticas e sociotécnicas antes de se propor a transposição de políticas, ou um seguimento acrítico das chamadas "melhores práticas" (Thomas; Dagnino, 2005; Stilgoe, Owen et al., 2013).

Por exemplo, em um estudo comparativo sobre dois exercícios de participação cidadã em nanotecnologia, Macnaghten e Guivant (2011) identificam como as atitudes dos públicos brasileiro e britânico são contrastantes no que toca à concepção sobre o papel da participação nos processos decisórios. Enquanto os participantes ingleses apresentaram concepções com maiores inclinações para a desconfiança e pessimismo para as implicações da nanotecnologia, os brasileiros foram mais receptivos a narrativas progressistas, apresentando muito menos criticismo em relação ao desenvolvimento tecnocientífico e, portanto, uma menor demanda para a transparência pública das decisões de 
investigação. Utilizando o conceito de "epistemologia cívica" de Jasanoff (2005) para "contextualizar as respostas públicas à ciência e tecnologia como cultural e historicamente situadas" (MacNaghten e Guivant 2011, p. 210), os autores concluem que a cultura política brasileira diverge bastante da britânica. No Brasil há uma menor demanda por mecanismos de participação individual para influenciar a política e decisões relacionadas com tecnologias emergentes, como os desenvolvidos no âmbito das políticas de nanotecnologia inglesas. Ainda que existam movimentos sociais fortes e bem organizados no país, existem questões sociais e políticas que se aparentam mais urgentes e prioritárias na arena pública (Hilgartner; Bosk, 1988) brasileira, isto é, não há a mesma receptividade e disposição por parte de porta-vozes desses movimentos sociais em participar de exercícios de diálogo.

Portanto, existem ainda outras barreiras que devem ser consideradas para a consolidação de práticas de inovação responsável em contextos socioculturais distintos dos verificados nos países centrais. Particularmente para o caso brasileiro, é preciso pensar os mecanismos de democratização para uma situação em que, conforme colocam Macnaghten e Guivant (2011), "nem os cientistas, nem os políticos, e muito menos o público apresentam o interesse em participar deste debate" (Macnaghten; Guivant, 2011, p. 218). Para tanto, faz-se necessário partir do reconhecimento de demandas específicas da região, que requerem escolhas teórico-metodológicas adequadas para o desenvolvimento sustentável e socialmente coeso da tecnociência em contextos periféricos (Dagnino; Fonseca, 2016). Acredita-se que contribuições profícuas possam ser desenvolvidas a partir da complementariedade entre algumas dessas abordagens e propostas oriundas da tradição dos ESCT da América Latina, o que ficou conhecido como Pensamento Latino Americano sobre Ciência, Tecnologia e Sociedade (PLACTS), como por exemplo a plataforma cognitiva que une desenvolvimento técnico científico e economia solidária que ficou conhecida como Tecnologia So- 
cial (Dagnino, 2014). Nesse sentido, esse autor tem apresentado em outros "fora" de discussão a sua proposta de Nanotecnologia Social (Fonseca; Pereira, 2014), como uma possível agenda de pesquisa que possa contribuir para a coprodução responsável da nanotecnologia no contexto sociotécnico brasileiro.

\section{Conclusão}

Apesar de variações em forma e dimensão, as iniciativas voltadas para uma inovação responsável aqui discutidas partem de pressupostos comuns, em torno do que foi denominado como "Governação Antecipatória da Nanotecnologia" (Barben et al., 2008). Basicamente, são propagadas a implementação de mecanismos que busquem uma ampliação do diálogo e da participação democrática na tomada de decisões tecnocientíficas, exercícios de avaliação ex ante, isto é, que procurem antecipar e avaliar, desde um estágio prematuro de desenvolvimento, possíveis implicações sociais, éticas, econômicas ou ambientais, e a integração das ciências sociais e humanas nos processos de investigação e desenvolvimento. Nesse sentido, a nanotecnologia tem servido de palco para uma inédita chamada para a colaboração ou integração das ciências sociais e naturais para a promoção de um desenvolvimento responsável.

Ainda que se reconheça uma apropriação ou distorção política do discurso vinculado à inovação responsável, é preciso também reconhecer que, no plano conceitual e metodológico, algumas abordagens merecem ser compreendidas e consideradas no debate sobre uma governança apropriada da nanotecnologia no Brasil. Isso não implica em simplesmente importar modelos de participação ou avaliação de tecnologias que podem se mostrar inviáveis e mesmo desnecessários para as prioridades brasileiras, mas sim trazê-las para o conhecimento dos públicos interessados, para que se possa democraticamente coproduzir políticas públicas que possibilitem a emergência de inovações nanotec- 
nológicas seguras, ambientalmente sustentáveis, mas sobretudo socialmente inclusivas.

\section{Ref erências}

BARBEN, Daniel. Analyzing acceptance politics: Towards an epistemological shift in the public understanding of science and technology. Public Understanding of Science 2010 19: 274 originally published online 26 June 2009, v. 19, n. 3, p. 274-292, 2010.

BARBEN, Daniel et al. Anticipatory Governance of Nanotechnology: Foresight, Engagement and Integration. In: HACKETT, E. J.; AMSTERDAMSKA, O., et al (Ed.). Handbook of Science and Technology Studies. Third. Cambridge, London: The MIT Press, 2008. cap. 38, p.979-1000.

BECK, Ulrich. Risk Society: Towards a New Modernity. London: Sage, 1992. BIJKER, W. E. . Of bicycles, bakelites, and bulbs: toward a theory of sociotechnical change. Cambrigde, MA: The MIT Press, 1995.

BUTTS, C.F. Philosophy of Science, v. 15, n. 2, 1948.

CALLON, M.; LASCOUMES, P.; BARTHE, Y.. Acting in an Uncertain World: An Essay on Technical Democracy. Cambridge, MA: The MIT Press, 2009.

COLLINGRIDGE, David. The Social Control of Technology. New York St. Martin's Press, 1980.

COPE, David. Parliaments and Technology Assessment. Minerva, v. 40, n. 4, p. 421-424, 2002. ISSN 0026-4695.

COZZENS, S.; KALLERUD, E.; PEREIRA, T. S. The Social Cohesion Policy Paradigm in Science and Technology Policy. in Prime-Latin America Conference. Mexico City September 24-26, 2008.

DAGNINO, Renato. A comunidade de pesquisa dos países avançados e a elaboração da política de ciência e tecnologia. Revista Brasileira de Ciências Sociais, v. 21, p. 191-201, 2006.

. Ciência e tecnologia no Brasil: o processo decisório e a comunidade de pesquisa. Campinas: Editora da Unicamp, 2007.

A construção do Espaço Ibero-americano do Conhecimento, os estudos sobre ciência, tecnologia e sociedade e a política científica e tecnológica. Revista CTS, v. 4, n. 12, 2009. 
Tecnologia Social: contribuições conceituais e metodológicas. Florianópolis: Inuslar e Campina Grande: EDUEPB: 2014.

DAGNINO, R.; FONSECA, P. F. C. Estudos Sociais da Ciência e Tecnologia na Ibero-américa: uma proposta taxonômica. In: CASAS, R. e MERCADO, A. (Ed.). Mirada iberoamericana a las políticas de ciencia, tecnología e innovación : perspectivas comparadas. Buenos Aires: CLACSO, 2016.

DAVID, Paul A. Clio and the Economics of QWERTY. The American Economic Review, v. 75, n. 2, 1985.

DAVIES, S.; MACNAGHTEN, P.; KEARNES, M. Reconfiguring Responsibiliy: Lessons for Public Policy (Part 1 of the report on Deepening Debate on Nanotechnology). Durham: Durham University, 2009.

DIAS, Rafael. A trajetoria da política cientifica e tecnologica brasileira : um olhar a partir da analise de politica. (PhD). Instituto de Geociências - Programa de Pós-Graduação em Política Científica e Tecnológica, Unicamp, Campinas, 2009.

DOSI, Giovanni. Technological paradigms and technological trajectories: A suggested interpretation of the determinants and directions of technical change. Research Policy, v. 11, p. 147-162, 1982.

DOUBLEDAY, R.; VISEU, A. Questioning Interdisciplinarity: What roles for laboratory based social science? In: F, K. K. A. W. (Ed.). Nano Meets Macro: Social perspectives on nanoscale sciences and technologies. Singapore: Pan Stanford Publishing, 2010.

EUROPEAN COMMISSION. Science and Society Action Plan. Luxembourg: Office for Official Publications of the European Communities, 2002.

Towards a European Strategy for Nanotechnology. Brussels: European Commission, 2004.

Nanosciences and Nanotechnologies: An action plan for Europe 2005-2009. Brussels: European Commission, 2005.

FISHER, E.; MAHAJAN, R. L. Contradictory intent? US federal legislation on integrating societal concerns into nanotechnology research and development. Science and Public Policy, v. 33, n. 1, p. 5-16, 2006.

FISHER, E.; MAHAJAN, R. L.; MITCHAM, C.. Midstream Modulation of Technology: Governance From Within. Bulletin of Science, Technology \& Society, v. 26, n. 6, p. 485-496, December 1, 2006.

FONSECA, P. F. C. A virada deliberativa nos Estudos Sociais da Ciência e Tecnologia e seus reflexos para novos regimes de inovação. Liinc em Revista, v. 8, n. 1, p. 151-164, 2012. 
FONSECA, P. F. C.; PEREIRA, T. S.. The Governance of Nanotechnology in the Brazilian Context: entangling approaches. Technology in Society, v. 37, p. 16$27,2014$.

FORSBERG, Ellen-Marie et al. Integrated assessment of emerging science and technologies as creating learning processes among assessment communities. Life Sciences, Society and Policy v. 12, n. 9, 2016.

FREEMAN, C. Technological infrastructure and international competitiveness. Industrial and Corporate Change, v. 13, n. 3, p. 541-569, 2004[1984].

FREEMAN, C.; SOETE, L. London: Continuum, 2000.

GALSTON, Arthur W. Science and Social Responsibility: A Case History. Annals of the New York Academy of Sciences, v. 196, n. Article 4, p. 223-235, 1972.

GROVE-WHITE, R.; MACNAGHTEN, P.; WYNNE, B. Wising Up: The public and new technologies. Lancaster University. Lancaster. 2000

GUSTON, D. H.; SAREWITZ, D. Real Time Technolgy Assessment. Technology in Society, v. 23, n. 4, p. 1-17, 2001.

HAGENDIJK, R.; HEALEY, P.; PEREIRA, T. S.. Researching Inequality through Science and Technology - ResIST Final Report. 2009

HILGARTNER, S.; BOSK, C. The rise and fall of social problems: A public arenas model. American Journal of Sociology v. 94, n. 1, p. 53-78, 1988.

HOVEN, JEROEN VAN DEN et al. Options for Strengthening Responsible Research and Innovation. Luxembourg: European Commission, 2013.

INVERNIZZI, Noela. Science Policy and Social Inclusion: Advances and Limits of Brazilian Nanotechnology Policy. In: COZZENS, S. E. e WETMORE, J. M. (Ed.). Yearbook of Nanotechnology in Society: Nanotechnology and the Challenges of Equity, Equality and Development. Dordrecht Heidelberg London New York: Springer, v.Volume 2, 2011. cap. 18, p.291-318.

IRWIN, Alan. The Politics of Talk. Social Studies of Science, v. 36, n. 2, p. 299320, April 1, 20062006.

IRWIN, A.; MICHAEL, M. Science, social theory and public knowledge. Maidenhead Philadelphia: Open University Press, 2003.

JASANOFF, Sheila. Civilization and madness: the great BSE scare of 1996. Public Understanding of Science, v. 6, n. 3, p. 221-232, July 1, 1997.

Technologies of Humility: Citizen Participation in Governing Science. Minerva, v. 41, p. 223-244, 2003. 
Designs on Nature: Science and Democracy in Europe and The United States. New Jersey: Princeton Universtiy Press, 2005.

JASANOFF, S.; KIM, S. Containing the Atom: Sociotechnical Imaginaries and Nuclear Power in the United States and South Korea. Minerva v. 47, p. 119-146, 2009.

JOLY, Pierre-Benoit. Innovation " responsable » et développement durable: Produire la légitimité des OGM et de leur monde. Fututibles, n. 383, p. 89-110, 2012.

JOLY, P.; KAUFMANN, A. Lost in Translation? The Need for Upstream Engagement with Nanotechnology on Trial. Science as Culture, v. 17, n. 3, p. 225-247, 2008.

KLÜVER, L et al. EUROPTA European Participatory Technology Assessment: Participatory Methods in Technology Assessment and Technology Decision-Making. Copenhagen K, Denmark, 2000.

KOOIMAN, Jan. Governing as Governance. London, Thousand Oaks, New Delhi: Sage Publications, 2003.

LAURENT, Brice. Technologies of Democracy: Experiments and Demonstrations. Science and Engineering Ethics, v. 17, p. 649:666, 2011.

LEWENSTEIN, Bruce. The meaning of 'public understanding of science' in the United States after World War I1. Public Understanding of Science, v. 1, n. 1, p. 45-68, 1992.

LEWENSTEIN, Bruce V. Models of public communication of science and technology, 2003.

LINSINGEN, I.V.; CASSIANI, S. Educação CTS em perspectiva discursia: contribuições dos estudos sociais da ciência e da tecnologia. In: CASSIANI, S.;SILVA, H. C. D., et al (Ed.). Olhares para o ENEM na Educação Científica e Tecnológica. Araraquara - SP: Junqueira \& Martin, 2013.

LUNDVALL, Bengt-Ake. Introduction to 'Technological infrastructure and international trade' by Christopher Freeman. Industrial and Corporate Change, v. 13, n. 3, p. 531-539, 2004.

LUNDVALL, B.; BORRAS, S.. Science, Technology and Innovation Policy. In: JAN FAGERBERG, D. C. M., RICHARD R. N. (Ed.). The Oxford Handbook of Innovation. New York: Oxford University Press, 2005.

MACNAGHTEN, P.; GUIVANT, J. S. Converging citizens? Nanotechnology and the political imaginary of public engagement in Brazil and the United Kingdom. Public Understanding of Science, v. 20, n. 2, p. 207-220, March 1, 2011. 
MACNAGHTEN, P.; KEARNES, M.; WYNNE, B. Nanotechnology, Governance, and Public Deliberation: What Role for the Social Sciences? Science Communication, v. 27, n. 2, p. 1-24, 2005.

MARRIS, C; JOLY, P.; RIP, A. Interactive Technology Assessment in the Real World: Dual Dynamics in an iTA Exercise on Genetically Modified Vines. Science, Technology \& Human Values, v. 33, n. 1, p. 77-100, January 1, 2008.

NELKIN, D; FALLOWS, S. The Evolution of the Nuclear Debate: The Role of Public Participation. Annual Review of Energy, v. 3, n. 1, p. 275-310, 1978.

OWEN, R.; GOLDBERG, N.. Responsible Innovation: A Pilot Study with the U.K. Engineering and Physical Sciences Research Council. Risk Analysis, v. 30, n. 11, p. 1699-1707, 2010.

OWEN, R.; MACNAGHTEN, P.; STILGOE, J. Responsible research and innovation: From science in society to science for society, with society. Science and Public Policy, v. 39, n. 6, p. 751-760, December 1, 2012.

OWEN, R. et al. A Framework for Responsible Innovation. In: R. OWEN, J. B. M. H. (Ed.). Responsible Innovation: Managing the Responsible Emergence of Science and Innovation in Society. Chichester, UK: John \& Wiley Sons, 2013. p. 27-50.

PATRA, Debasmita. Responsible Development of Nanoscience and Nanotechnology: Contextualizing Socio-Technical Integration into the Nanofabrication Laboratories in the USA. Nanoethics, v. 5, p. 143-157, 2011.

PAVITT, Keith. Sectoral patterns of technical change: Towards a taxonomy and a theory. Research Policy, n. 13, p. 343-373, 1984.

PINCH, T.; BIJKER, W.. The Social Construction of Facts and Artifacts: Or How the Sociology of Science and the Sociology of Technology Might Benefit Each Other. In: BIJKER, W.; HUGHES, T., et al (Ed.). The Social Construction of Technological Systems: MIT Press, 1989. p.17-50.

POLANYI, Michael. The Republic of Science: Its Political and Economic Theory. Minerva, v. I, n. 1, p. 54-73, 1962.

RESPONSIBLE NANOCODE FOUNDING PARTNERS. The Responsible NanoCode. The Royal Society; Insight Investiment Management (Global) Limited, et al 2008.

RIBEIRO, B. E.; SMITH, R. D. J.; MILLAR, K. A Mobilising Concept? Unpacking Academic Representations of Responsible Research and Innovation. Science and Engineering Ethics, v. 2016 Mar 8. [Epub ahead of print], 2016. 
RIP, Arie. Introduction of New Technology; Making Use of Recent Insights from Sociology and Economics of Technology. Technology analysis and strategic management, v. 7, n. 4, p. 417-431, 1995.

Folk Theories of Nanotechnologists. Science as Culture, v. 15, n. 4, p. 349 - 365, 2006.

RIP, A.; KULVE, H. . Constructive Technology Assessment and Socio-Technical Scenarios. In: FISHER, E.;SELIN, C., et al (Ed.). The Yearbook of Nanotechnology in Society, Volume I: Presenting Futures: Springer Netherlands, v.1, 2008. p. 49-70. (Yearbook of Nanotechnology in Society).

ROBINSON, Douglas K. R. Co-evolutionary scenarios: An application to prospecting futures of the responsible development of nanotechnology. Technological Forecasting and Social Change, v. 76, n. 9, p. 1222-1239, 2009.

. Constructive Technology Assessment of Emerging Nanotechnologies Experiments in Interactions. (PhD). University of Twente, 2010.

ROCO, M. C. et al. Innovative and Responsible Governance of Nanotechnology for Societal Development. In: ROCO, M. C.; MIRKIN, C. A., et al (Ed.). Nanotechnology Research Directions for Societal Needs in 2020: Retrospective and Outlook. Boston, Berlin: Springer, 2010.

ROWE, G.; FREWER, L. J. Public Participation Methods: A Framework for Evaluation. Science, Technology Human Values, v. 25, n. 1, p. 3-29, 2000.

A Typology of Public Engagement Mechanisms. Science, Technology \& Human Values, v. 30, n. 2, p. 251-290, 2005.

ROYAL SOCIETY; ROYAL ACADEMY OF ENGENEERS. Nanoscience and nanotechnologies: opportunities and uncertainties. London: The Royal Society \& The Royal Academy of Engineering, 2004.

SANTOS, Boaventura de Sousa. Para além do pensamento abissal: das linhas globais a uma ecologia de saberes. In: SANTOS, B. D. S. e MENESES, M. P. (Ed.). Epistemologias do Sul. Coimbra: Almedina, 2010.

SANTOS JUNIOR, Jorge Luiz dos. Ciência do Futuro: A Comunidade de Pesquisa e o Ciclo da Política de Nanociência no Brasil. Instituto de Ciências Humanas e Naturais, Universidade Federal Rural do Rio de Janeiro, 2011.

SCHOMBERG, René Von. Towards Responsible Research and Innovation in the Information and Communication Technologies and Security Technologies Fields. Brussels: European Commission, 2011.

SCHOT, Johan. Towards New Forms of Participatory Technology Development. Technology Analysis \& Strategic Management, v. 13, n. 1, p. 39-52, 2001. 
SCHOT, J.; RIP, A.. The past and future of constructive technology assessment. Technological Forecasting and Social Change, v. 54, n. 2-3, p. 251-268, 1997.

SCHUMPETER, Joseph. The Creative Response in Economic History. The Journal of Economic History, v. 7, n. 2, p. 149-159, 1947.

SCLOVE, Richard E. Reinventing Technology Assessment. Issues in Science and Technology, 2010.

SHIVA, Vandana. Monocultures, Monopolies, Myths and the Masculinization of Agriculture. Development, v. 42, n. 2, p. 35-38, 1999.

STILGOE, J.; OWEN, R.; MACNAGHTEN, P. Developing a framework for responsible innovation. Research Policy, v. 42, n. 9, p. 1568-1580, 2013.

THE ROYAL SOCIETY OF LONDON. The Public Understanding of Science. London: Royal Society, 1985.

THOMAS, Hernan. Tecnologias para Inclusão Social e Políticas Públicas na América Latina. In: RTS, R. D. T. S. (Ed.). Tecnologias Sociais: Caminhos para a sustentabilidade. Brasília: RTS, 2009.

THOMAS, H.; DAGNINO, R.. Efectos de transducción: una nueva crítica a la transferencia acrítica de conceptos y modelos institucionales. Ciencia, Docencia y Tecnología, v. 16, n. 31, p. 9-46, 2005.

THORPE, Charles. Participation as Post-Fordist Politics: Demos, New Labour, and Science Policy. Minerva, v. 48, n. 4, p. 389-411, 2010.

UE. Conclusões da Presidência do Conselho de Lisboa. EUROPEU, C. Lisboa. 23 e 24 de Março 2000.

UK GOVERNMENT. UK Government's response to the Royal Society and Royal Academy of Engineering report Nanoscience and nanotechnologies: opportunities and uncertainties. London: UK Government, 2005.

VAN APELDOORN, Bastiaan. The Lisbon Agenda and the Legitimacy Crisis of European Socio-Economic Governance: the Future of 'Embedded Neo-Liberalism'. In: (Ed.). 4th Convention of the Central and Ealt European International Studies Association Tartu, Estonia: CEEISA, 2006.

VAN DOREN, D.; FORSBERG, E.; LINDNER, R. Are assessments responding to a dynamic environment? Evidence from four emerging techno-scientific domains. Science and Public Policy, v. 41, n. 3, p. 317-331, June 1, 2014.

VAN MERKERK, R. O.; ROBINSON, D. K. R. Characterizing the emergence of a technological field: Expectations, agendas and networks in Lab-on-a-chip technologies. Technology Analysis \& Strategic Management, v. 18, n. 3, p. 411428, 2006. 
VAN MERKERK, R. O.; VAN LENTE, H.. Tracing emerging irreversibilities in emerging technologies: The case of nanotubes. Technological Forecasting and Social Change, v. 72, n. 9, p. 1094-1111, 2005.

VELHO, Léa. Conceitos de Ciência e a Política Científica, Tecnológica e de Inovação. Sociologias, v. 13, n. 26, p. 128-153, 2011.

VIOTTI, Eduardo B. Brazil: From S\&T to innovation policy? The evolution and the challenges facing Brazilian policies for science, technology and innovation. VI Globelics Conference,. Mexico City 2008.

WHITMAN, Jim. The governance of nanotechnology. Science and Public Policy, v. 34, n. 4, 2007.

WILSDON, J.; WILLIS, R.. See-through Science: Why public engagement needs to move upstream. London: Demos, 2004.

WOOD, Fred B. Lessons in technology assessment : Methodology and management at OTA. Technological Forecasting and Social Change, v. 54, n. 2-3, p. 145-162, 1997.

WYNNE, Brian. Misunderstood misunderstanding: social identities and public uptake of science. Public Understanding of Science, v. 1, n. 3, p. 281-304, 1992.

Public engagement as a means of restoring public trust in science--hitting the notes, but missing the music? Community Genetics, v. 9, n. 3, p. 211-220, 2006.

Rationality and Ritual: Participation and Exclusion in Nuclear Decision-making. Lancaster: Rouledge, 2011. 